\title{
A MODEL OF $\gamma$-RAY SOURCES IN THE GALAXY
}

\author{
S. HAYAKAWA and Y. TANAKA
}

Dept. of Physics, Nagoya University, Nagoya, Japan

In a recent observation Clark et al. [1] have found that the intensity of $\gamma$-rays of energies around $100 \mathrm{MeV}$ is strong in the direction of the galactic plane; the observed intensity of the order of $10^{-4} \mathrm{~cm}^{-2} \mathrm{sec}^{-1} \mathrm{rad}^{-1}$ is greater by an order of magnitude than that predicted on the neutral pion decay hypothesis [2]. It should, however, be remarked that the theory is based on the assumption that cosmic rays are uniformly distributed in our galaxy. The assumption seems doubtful in view of the experimental result that the $\gamma$-ray intensity depends rather strongly on the galactic longitude.

If cosmic rays were uniform in the galactic disk, the intensity of $\gamma$-rays produced by neutral pion decays would have to be proportional to the column density of interstellar matter. Although the observed longitude dependence of the $\gamma$-ray intensity qualitatively shows such a tendency, it is much stronger than the longitude dependence of the column density of hydrogen atoms obtained from $21-\mathrm{cm}$ radio observations [3]. The

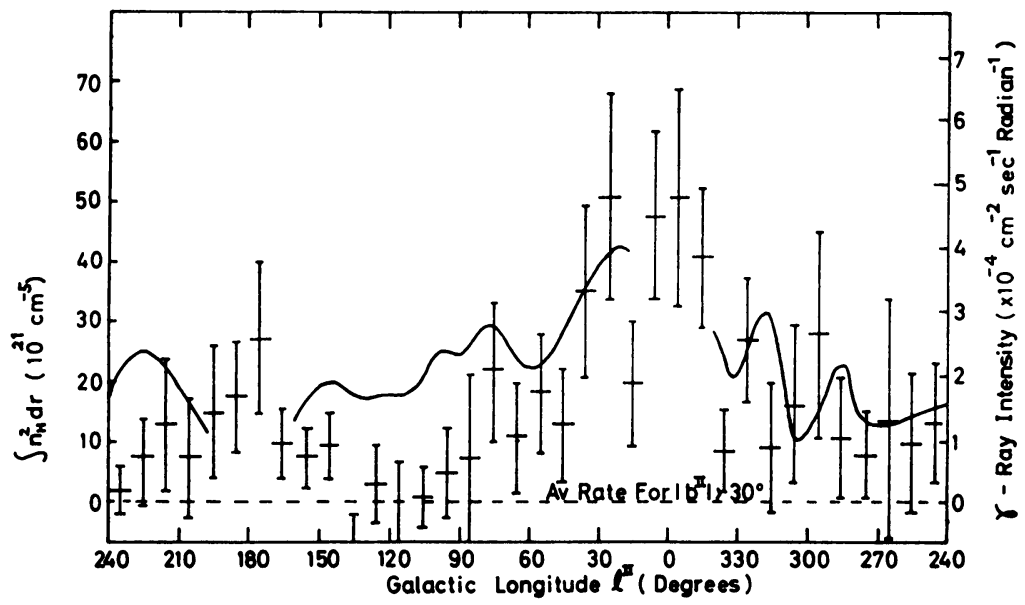

Fig. 1. Longitude dependences of the $\gamma$-ray intensity and the column square density of hydrogen atoms.

$\gamma$-ray intensity is better correlated with the column square density of hydrogen atoms,

$$
\left\langle n_{\mathrm{H}}^{2} R\right\rangle=\int n_{\mathrm{H}}^{2} \mathrm{~d} r
$$

as shown in Figure 1.

The correlation shown in Figure 1 seems to indicate that the density of $\gamma$-ray sources is proportional to the square density of the interstellar gas. The same relation has been 
suggested to hold also for X-ray sources, and the reason for this has been attributed to the fact that the star formation rate is proportional to the square density of gas and that most X-ray sources are associated with stars of ages younger than $10^{9}$ years [4]. The same argument may hold for $\gamma$-ray sources. It is thus assumed that most $\gamma$-ray sources are associated with supernova remnants, a majority of which belong to type II supernovae and are identified with galactic non-thermal radio sources. The radio intensity of these sources [5] is compared with the $\gamma$-ray intensity in each longitude range in Figure 2. The agreement between these two is fairly good, if a few strong radio sources such as Cas $\mathrm{A}$ and $\mathrm{Tau} \mathrm{A}$ are discarded.

The correlations shown above seem to suggest that the galactic $\gamma$-rays are produced mainly by neutral pion decays in supernova remnants. If this is the case, electrons are

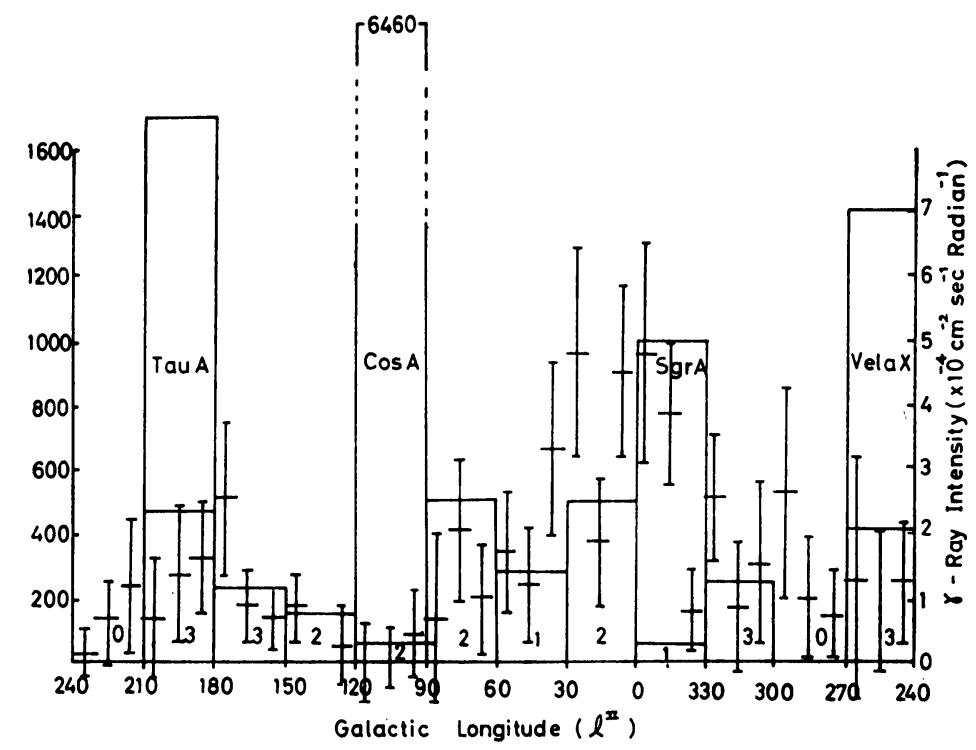

Fig. 2. Correlation between the $\gamma$-ray intensity and the radio intensity of supernova remnants. The numbers of supernova remnants adopted are indicated. Four strongest sources are separately indicated.

produced as well by charged meson decays and the radio waves emitted by these electrons would be too strong. This difficulty can be avoided, if strong magnetic fields are concentrated in an expanding shell rather than they are distributed uniformly in the whole volume of a remnant. This feature is in fact seen in Cygnus loop and other supernova remnants, and also is favored in the radio intensity distribution in the Crab Nebula. In what follows we shall demonstrate that this may be the case.

From Figure 2 we see that the intensity of $\gamma$-rays is about $J_{\gamma}=1 \times 10^{-4} \mathrm{~cm}^{-2} \mathrm{sec}^{-1}$ $\mathrm{rad}^{-1}$. This is related to the production rate at a source, $q_{\gamma}$, and the surface density, $D$, of the sources in the galactic disk of thickness, $d$, as

$$
J_{\gamma}=\frac{q_{\gamma} D}{4 \pi}\left[1+\ln \left(\frac{R_{\mathrm{m}}}{d / 2 b_{\mathrm{m}}}\right)\right],
$$


where the $\gamma$-rays coming from galactic latitudes within $\pm b_{\mathrm{m}}$ and distances within $R_{\mathrm{m}}$ are included. Since $b_{\mathrm{m}}=15^{\circ}[1]$ and $D=0.85 \mathrm{kpc}^{-2}$ [5], we obtain

$$
q_{\gamma} \simeq 3 \times 10^{39} \mathrm{sec}^{-1} \text {. }
$$

On the other hand, the radio yield at $1 \mathrm{GHz}$ is evaluated by reference to the compilation in [5] as

$$
Y \simeq 2 \times 10^{23} \mathrm{erg} \mathrm{sec}^{-1} \mathrm{~Hz}^{-1} .
$$

The radio yield can be accounted for in terms of synchrotron radiation of relativistic electrons in magnetic fields. The intensity of the electrons is assumed as $1 / K$ times the proton intensity. If the radio emitting region is restricted to the shell of thickness $\Delta r$, which is smaller than the radius of a supernova remnant, $r$, we obtain

$$
q_{\gamma} / Y \simeq 0.7 \times 10^{14} \mathrm{Kn}_{\mathrm{H}} \mathrm{H}^{-(1+\alpha)}(r / 3 \Delta r),
$$

where $n_{\mathrm{H}}$ is the hydrogen density in $\mathrm{cm}^{-3}, H$ is the magnetic field strength in $\mu$ gauss and $\alpha$ is the radio spectral index which is in most cases 0.7 . This is compared with the observed value of $1.5 \times 10^{16} \mathrm{erg}^{-1} \mathrm{~Hz}$. For a typical set of values, $K=10^{2}, n_{\mathrm{H}}=10$, and $H=10$, we find that $r / \Delta r=30$ can account for the observed ratio of $\gamma$-ray and radio intensities.

A question arises whether or not the value of $q_{\gamma}$ estimated in (3) is consistent with the intensity of electrons. Since there are about 600 radio emitting supernova remnants, the $\gamma$-ray production rate in our galaxy is estimated as

$$
Q_{\gamma} \approx 600 q_{\gamma} \approx 2 \times 10^{42} \mathrm{sec}^{-1} .
$$

This is compared with the intensity of electrons observed. A recent measurement of positrons with energies greater than $100 \mathrm{MeV}$ gives an intensity of about $1 \times 10^{-3} \mathrm{~cm}^{-2}$ $\mathrm{sec}^{-1} \mathrm{sr}^{-1}$ [6]. Since most of such low energy electrons from meson decays are positive and the electron spectrum tends to be flat below a few hundred $\mathrm{MeV}$, the total intensity of meson-decay electrons may not exceed $2 \times 10^{-3} \mathrm{~cm}^{-2} \mathrm{sec}^{-1} \mathrm{sr}^{-1}$. This would require that the electron production rate in our galaxy should be about $3 \times 10^{41} \mathrm{sec}^{-1}$, which is by an order of magnitude smaller than that given in (6), if the electrons were uniformly distributed in our galaxy and they were not subject to solar modulation. If, however, the solar modulation is appropriately taken into account [7], the interstellar intensity of electrons with energies around and below $100 \mathrm{MeV}$ expected from the production rate (6) is not inconsistent with the observed intensity of positions. It should also be remarked that the former is neither inconsistent with the general galactic radio emission.

\section{References}

[1] Clark, G. W., Garmire, G. P., and Kraushaar, W. L.: 1968, Astrophys. J. 153, L203.

[2] Hayakawa, S., Okuda, H., Tanaka, Y., and Yamamoto, Y.: 1964, Prog. Theor. Phys. Suppl. No. $30,153$.

[3] Kerr, F. J.: 1962, Monthly Notices Roy. Astron. Soc. 123, 327.

[4] Fujimoto, M., Hayakawa, S., and Kato, T.: 1969, Astrophys. Space Sci. 464.

[5] Poveda, A. and Woltjer, L.: 1968, Astron. J. 73, 65.

[6] Fanselow, J. L., Hartman, R. C., Hildebrand, R. H., and Meyer, P.: 1968.

[7] Webber, W. R.: 1968, Aust. J. Phys. 21, 845. 